\title{
Studies of hadronic $B$ decays with early LHCb data
}

\author{
Aurélien Martens ${ }^{* \dagger}$ \\ LAL, Université Paris-Sud, CNRS/IN2P3, Orsay, France \\ E-mail: martenselal.in2p3.fr
}

Hadronic $B$ decays offer rich opportunities for $C P$-violation studies. Decays of the type $B \rightarrow$ $D X$, where $D$ represents a charmed meson $\left(D^{0}, D^{(*)+}\right.$ or $\left.D_{s}^{+}\right)$, allow for a theoretically clean determination of the CKM triangle angle $\gamma$ which provides a Standard Model benchmark of this quantity, robust against new physics effects. Decays into charmless final states, on the other hand, in general receive significant contributions from loop diagrams and are susceptible to the effects of new heavy particles. The trigger schemes of LHCb, and the particle identification provided by its RICH system, give the experiment high sensitivity to these decays. Signals will be shown from the present LHC run, and the measurement program with the 2010-2011 data set will be discussed.

Workshop on Discovery Physics at the LHC-Kruger 2010

December 05-10, 2010

Kruger National Park, Mpumalanga, South Africa

\footnotetext{
* Speaker.

On behalf of the LHCb Collaboration.
} 


\section{Present status of the CKM matrix}

A decade after the beginning of data taking at $B$-factories, the sides and angles of the unitarity triangle (UT) of the Cabibbo-Kobayashi-Maskawa (CKM) matrix [1] are measured at a level better than $10 \%$ except the $\left|V_{u b}\right|$ side and the angle $\gamma$. There are different approaches to interpret the combination of measurements made by the global fits of the CKM matrix [2,3]. One of these is to check the consistency of the UT, but no unambiguous sign of discrepancy has been observed so far. For instance the prediction of $\gamma$ by the global fits leads to $67.2^{\circ} \pm 3.9^{\circ}$ (CKM fitter) or $69.6^{\circ} \pm 3.1^{\circ}$ (UT fit) depending on the statistical approach. A combination of (statistically limited) direct measurements of $\gamma$ provides $71^{\circ} \pm 23^{\circ}$ (CKM fitter) or $74^{\circ} \pm 11^{\circ}$ (UT fit). This shows the interest of measuring $\gamma$ with increased statistics, which can be performed at $\mathrm{LHCb}$.

\section{Measurements of $\gamma$}

Precise measurements of $\gamma$ would thus allow for precise consistency check of the CKM matrix paradigm. Another important feature of the angle $\gamma$ is that it can be measured by three independent classes of methods, allowing for direct and mixing induced induced $\mathrm{CP}$-violation measurements which are differently sensitive to New Physics effects. A comparison of these determinations will provide additional constraints on New Physics parameters.

\subsection{Time integrated $\gamma$ from trees}

Both charmed $B^{ \pm}$decays and self-tagging charmed $B^{0}$ decays are pure tree decays and allow for a benchmark measurement of $\gamma$ in the Standard Model, since they are expected to be free from contributions of New Physics. These decays are extremely clean theoretically with an irreducible uncertainty of $\delta \gamma / \gamma \simeq 10^{-6}$ [4]. A comparable statistical uncertainty would only be reached with $\mathscr{O}\left(10^{18}\right)$ pairs of $B$ mesons produced, which is far beyond the scope of LHCb or a super $b$-flavour factory.

The measurement is performed taking advantage of the interference of $b \rightarrow c$ and $b \rightarrow u$ transitions, the latter being sensitive to the $C P$-angle $\gamma$. Thus there is a compromise to find between the absolute rate of the decay and the relative size of its interference term that drives the sensitivity to $\gamma$. Usually considered as the most powerful are the decays $B^{ \pm} \rightarrow D K^{ \pm}$and $B^{0} \rightarrow D K^{* 0}$ for which the branching fractions are $\mathscr{B}\left(B^{+} \rightarrow \bar{D}^{0} K^{+}\right)=(3.7 \pm 0.3) \cdot 10^{-4}$ and $\mathscr{B}\left(B^{0} \rightarrow \bar{D}^{0} K^{* 0}\right)=$ (4.2 \pm 0.6$) \cdot 10^{-5}$ and the ratios of $b \rightarrow u$ to $b \rightarrow c$ amplitudes (that gives the size of the interference term and hence drives the sensitivity to $\gamma$ ) are $r_{B^{ \pm} \rightarrow D K^{ \pm}}=0.11 \pm 0.02[3]$ and $r_{B^{0} \rightarrow D K^{* 0}}=0.26 \pm 0.08$ [5], leading to similar sensitivity to $\gamma$.

Different approaches exist for the reconstruction of a $D$ meson $^{1}$. The Gronau-London-Wyler [6] strategy exploits $D$ decays in $C P$-eigenstates. Thus the decays of $D^{0}$ and $\bar{D}^{0}$ in the same final state have the same branching fractions in absence of $C P$-violation and no additional unknown is added in the extraction. This method has been extended to non $C P$-eigenstates decay channels common to $D^{0}$ and $\bar{D}^{0}$ in the so-called Atwood-Dunietz-Soni method [7]. In this case, two unknowns are added: the ratio of branching fractions of the $D^{0}$ decay to a given final state $f$ with respect to

\footnotetext{
${ }^{1} D$ represents either a $D^{0}$ or a $\bar{D}^{0}$ meson.
} 
the $\bar{D}^{0}$ decay to the same final state and a difference of strong phases. These additional parameters can be constrained by $\psi(3770) \rightarrow D \bar{D}$ data from CLEO or BES, resulting in a constant number of unknowns for a increase of observables. Finally the use of multibody $D$ decays is advantageous to recover some statistical power by using Dalitz plot studies of the $D$ decays in the Giri-GrossmanSoffer-Zupan [8] approach. Such latter measurements have provided so far the most statistically sensitive measurements of $\gamma$ at the $B$-factories.

Many final states are under study at LHCb, among them $K^{+} K^{-}, \pi^{+} \pi^{-}$(GLW), $K^{\mp} \pi^{ \pm}, K^{\mp} \pi^{ \pm} \pi^{0}$, $K^{\mp} \pi^{ \pm} \pi^{ \pm} \pi^{\mp}$ (ADS), $K_{\mathrm{S}}^{0} \pi^{ \pm} \pi^{\mp}, K_{\mathrm{S}}^{0} K^{ \pm} K^{\mp}$ (GGSZ). Interesting extensions to $B^{0} \rightarrow D K \pi$ or untagged $B_{s}^{0} \rightarrow D \phi$ can also be used at LHCb. A combination of all these measurements provides consistency checks as well as the best overall sensitivity to $\gamma$.

\subsection{Time-dependent $\gamma$ from trees}

Another path to extract $\gamma$, up to a mixing phase that is known by precise and model-independent mixing measurements (already well measured in the $B^{0}$ sector and will be precisely known thanks to $B_{s}^{0} \rightarrow J / \psi \phi$ decays in the $B_{s}^{0}$ sector), exploits time-dependent charmed $B$ decays [9]. This additional benchmark measurement could eventually be combined with the time-integrated measurements to provide a precise reference for New Physics tests.

The eight inherent ambiguities in the extraction of the $C P$-angle are resolved in the $B_{s}^{0} \rightarrow$ $D_{s}^{ \pm} K^{\mp}$ channel (except an irreducible 180 degrees ambiguity) thanks to the non-vanishing lifetime difference $\Delta \Gamma_{s}$ and exploiting the $B_{s}^{0} \rightarrow D_{s}^{ \pm} \pi^{\mp}$ mode. This channel is expected to be unique to LHCb. Despite a branching fraction of $\mathscr{B}\left(B_{s}^{0} \rightarrow D_{s}^{ \pm} K^{\mp}\right)=(3.0 \pm 0.7) \cdot 10^{-4}$ the ratio of $b \rightarrow u$ to $b \rightarrow c$ amplitudes is of $x_{B_{s}^{0} \rightarrow D_{s}^{ \pm} K^{\mp}} \simeq 0.4$ which allow for an easier extraction from data than for $B^{0} \rightarrow D^{ \pm} \pi^{\mp}$, for which $\mathscr{B}\left(B^{0} \rightarrow D^{ \pm} \pi^{\mp}\right)=(2.7 \pm 0.1) \cdot 10^{-3}$ and $x_{B^{0} \rightarrow D^{ \pm} \pi^{\mp}} \simeq 0.02$ (which is too small to be extracted directly from data). This latter can still be used to extract $\gamma$, but the ambiguities are not well resolved in this case. These two problems (remaining ambiguities and small interference term) can be worked out by use of an U-spin analysis combining the two sensitive channels $B_{s}^{0} \rightarrow D_{s}^{ \pm} K^{\mp}$ and $B^{0} \rightarrow D^{ \pm} \pi^{\mp}$.

\section{$2.3 \gamma$ from loops}

A complementary way, sensitive to New Physics effects, to extract $\gamma$ consists of measuring the $C P$ violation in $B^{0} \rightarrow h^{ \pm} h^{\prime} \mp$ decays, where $B$ represent either a $B^{0}$ or a $B_{s}^{0}$ and $h^{\left({ }^{\prime}\right)}$ either a $K$ or a $\pi$ [10]. These six decays have different contributions from Tree, Penguin, Penguin Annihilation, Colour-suppressed Electroweak Penguin and Exchange topologies, summarized in [11]. $\gamma$ is extracted from the $C P$-asymmetries in $B^{0} \rightarrow \pi^{+} \pi^{-}$and $B_{s}^{0} \rightarrow K^{+} K^{-}$using U-spin symmetry to reduce the number of unknowns. The validity of this assumption is checked using $B_{s}^{0} \rightarrow K \pi$ and $B^{0} \rightarrow K \pi$ and the size of Penguin Annihilation (PA) and Exchange (E) contributions, is probed by the $B^{0} \rightarrow K^{+} K^{-}$and $B_{s}^{0} \rightarrow \pi^{+} \pi^{-}$channels that are only sensitive to these topologies.

Direct and mixing induced $C P$-violation measurements in $B^{0} \rightarrow \pi^{+} \pi^{-}$and $B_{s}^{0} \rightarrow K^{+} K^{-}$allow for $\gamma$ extraction, under $\mathrm{U}$-spin assumption and using external measurements of mixing phases. The resulting $\gamma$ measurement, compared with the two tree level measurements of $\gamma$, can then provide a useful probe of New Physics. 


\section{The LHCb detector}

The $\mathrm{LHCb}$ detector [12] is a single arm spectrometer designed to accurately measure decay products of $B$ and $D$ mesons for precision measurements at the LHC. The commissioning of the detector and the performance reached with the first data is summarized in [13, 14]. Two key ingredients for the hadronic decays are the ability to trigger on and to disentangle different modes.

\subsection{Trigger}

The LHCb trigger system is based on a fast hardware decision (L0) on the transverse energy of hadrons, electrons, photons and muons to remove most of the non- $b$ events. A L0 hadron threshold of $3.6 \mathrm{GeV}$ has been used in the most significant period of data taking. The L0 has already been demonstrated to provide $300 \mathrm{kHz}$ of events and is expected to deliver $1 \mathrm{MHz}$ to the High Level Trigger (HLT) in 2011. The first layer of the software trigger (HLT1) reconstructs primary vertices (PVs) and tracks, looking for typical high $p_{T}$ and high impact parameter (IP) clean tracks [15]. The output rate is found to be quite robust with respect to the pile-up, saturating around $30 \mathrm{kHz}$ for an input rate of $1 \mathrm{MHz}$. The HLT2 is based on an inclusive approach [16] to look for hadronic $B$ decays. A $B$ candidate, with a $B$ mass corrected for missing transverse momentum using the constraint of the flight direction in the $4-7 \mathrm{GeV} / c^{2}$ range, is selected using either two, three or four tracks. This typically gives a HLT2 efficiency above $80 \%$ for most of the channels.

\subsection{Particle Identification}

The LHCb detector is designed to perform good $\pi$ versus $K$ (as well as $p, e$ and $\mu$ ) separation over a wide range of momentum thanks to two dedicated Ring Imaging Cherenkov (RICH) systems with different radiators. The RICH1 is located close to the Vertex Locator (VELO, a silicon strip detector for precise reconstruction of tracks close to the primary vertex) and uses aerogel and $\mathrm{C}_{4} \mathrm{~F}_{10}$ as radiators, allowing for $\pi$ versus $K$ separation in the $2-60 \mathrm{GeV} / c$ momentum range. Additional separation is provided by the RICH2 up to $100 \mathrm{GeV} / c$ thanks to a $\mathrm{CF}_{4}$ radiator. First valuable experience, understanding of alignment and calibration of the particle identification has been obtained in 2010 allowing for good $\pi$ versus $K$ separation and results relatively close to expectations.

\section{Results and perspectives}

One month after the end of $p p$ data taking at $\mathrm{LHCb}$, using a dataset corresponding to an integrated luminosity of $35 \mathrm{pb}^{-1}$ out of which approximately $90 \%$ has been recorded in the last month, $\mathrm{LHCb}$ is able to show its first invariant mass peaks of hadronically triggered channels. Some selected signals are presented here.

\subsection{Time integrated $\gamma$ from trees}

First signals in $B^{ \pm} \rightarrow D \pi^{ \pm}$with $C P$-eigenstates and three body $K_{\mathrm{S}}^{0} h h D$ decays have been observed (figure 1) as well as $B^{ \pm} \rightarrow D K^{ \pm}$with $D^{0} \rightarrow K^{-} \pi^{+}$(figure 2). Given the lower branching fraction in $B^{0} \rightarrow D K^{* 0}$, this mode is more difficult to observe but, another pure colour suppressed mode, $B^{0} \rightarrow D^{0} \rho^{0}$ is observed (figure 2). This may allow to obtain first evidence or first observation 

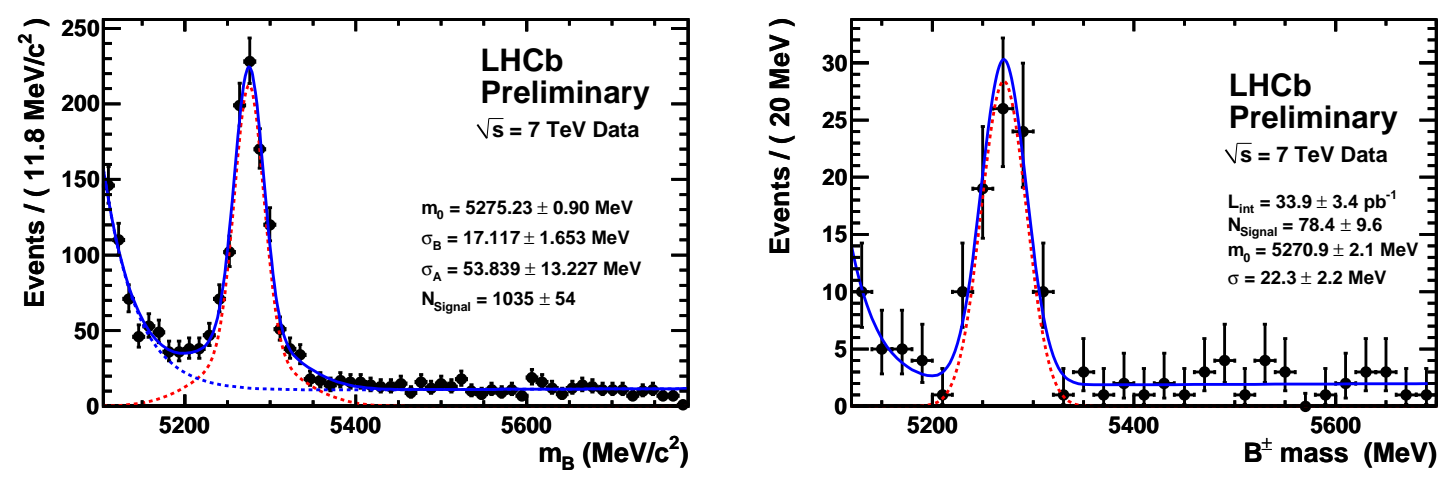

Figure 1: $B^{ \pm} \rightarrow D \pi^{ \pm}$with $D^{0} \rightarrow K^{-} K^{+}$(left) and with $D^{0} \rightarrow K_{\mathrm{S}}^{0} K^{-} K^{+}$(right) in $35 \mathrm{pb}^{-1}$ of LHCb data.
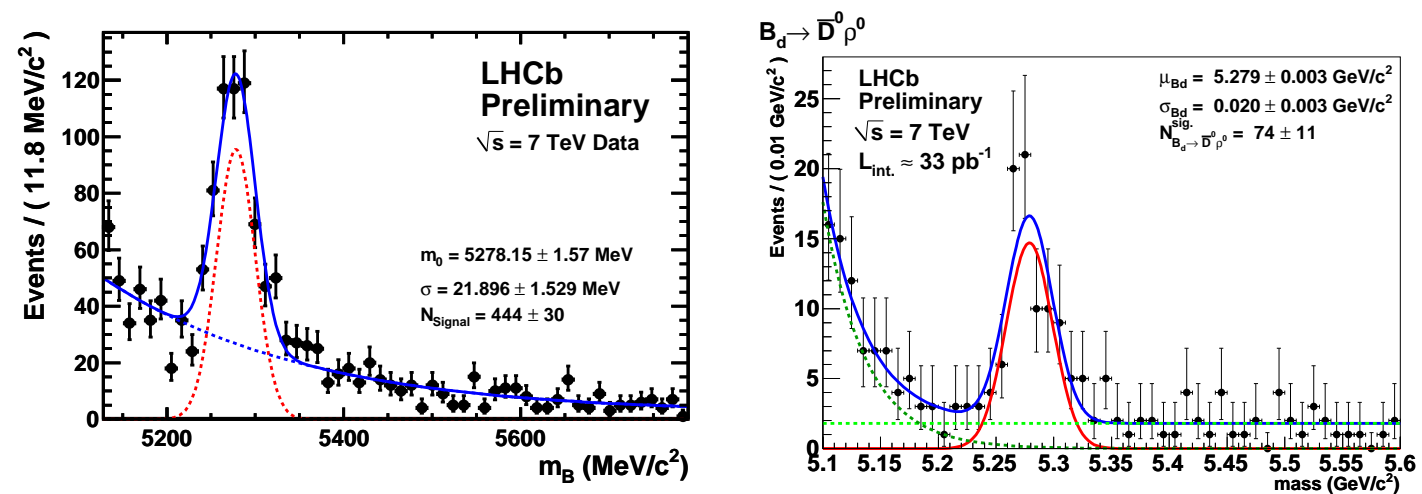

Figure 2: $B^{ \pm} \rightarrow D K^{ \pm}$(left) and $B^{0} \rightarrow D^{0} \rho^{0}$ (right) with $D^{0} \rightarrow K^{-} \pi^{+}$in $35 \mathrm{pb}^{-1}$ of LHCb data.

of $\bar{B}_{s}^{0} \rightarrow D^{0} K^{* 0}$ with $D^{0} \rightarrow K^{-} \pi^{+}$. One can also extrapolate that LHCb is on target to provide competitive measurements of $C P$ asymmetries with current best measurements both in $C P$-eigenstates and ADS analyses by the end of the next long period of data taking assuming at least $1 \mathrm{fb}^{-1}$ of integrated luminosity. Depending on the 2011-2012 data taking conditions, a combined sensitivity to $\gamma$ of the order of $10^{\circ}-15^{\circ}$ could be reached.

\subsection{Time-dependent $\gamma$ from trees}

Clean signals for time dependent measurements are also observed in $B_{s}^{0} \rightarrow D_{s}^{ \pm} \pi^{\mp}$ with $D_{s}^{+} \rightarrow$ $\phi \pi$ (figure 3). Additional channels for time dependent measurements of $\gamma$ could be used (for instance the six body final state $B^{0} \rightarrow D \pi \pi \pi$, figure 3). With the already accumulated data, a potential and important result that could be obtained from these decays is a measurement of $f_{s} / f_{d}$. An $10^{\circ}-15^{\circ}$ sensitivity on $\gamma$ could be obtained in 2011-2012, independent from the time-integrated measurements.

\section{$4.3 \gamma$ from loops}

First signs of asymmetry, that still need to be properly calibrated to quote values for $C P$ violation, have been observed in the first look at the 2010 data. Statistical uncertainties are $\simeq 0.04$ and 

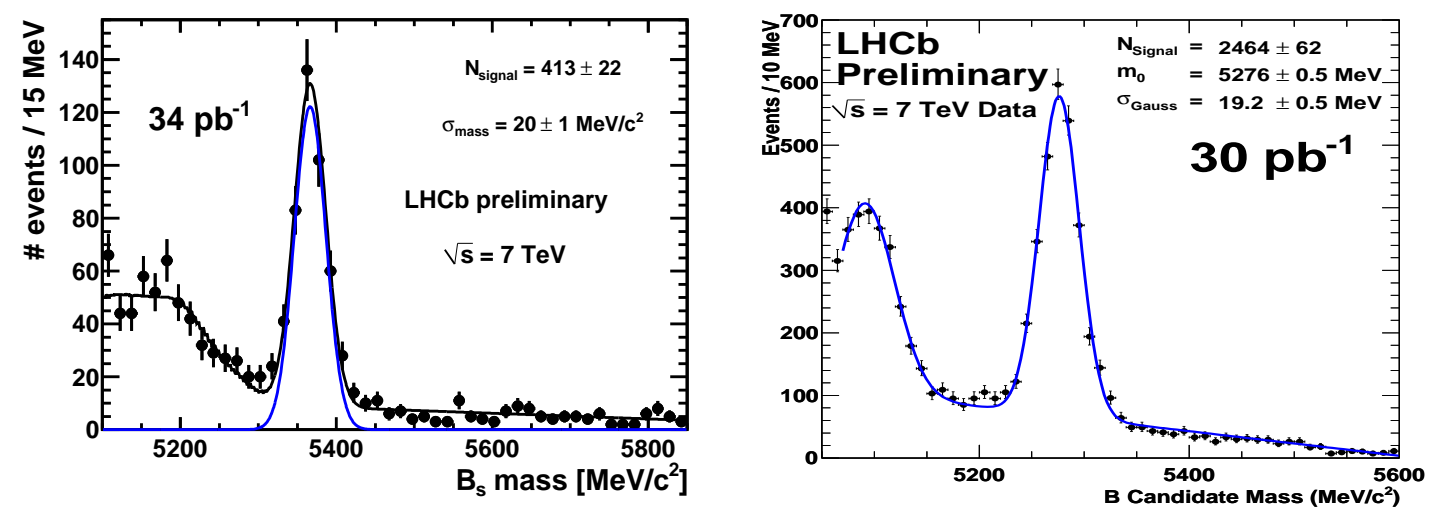

Figure 3: $B_{s}^{0} \rightarrow D_{s}^{ \pm} \pi^{\mp}$ with $D_{s}^{+} \rightarrow \phi \pi$ (left) and $B^{0} \rightarrow D \pi \pi \pi$ (right) in $35 \mathrm{pb}^{-1}$ of LHCb data.
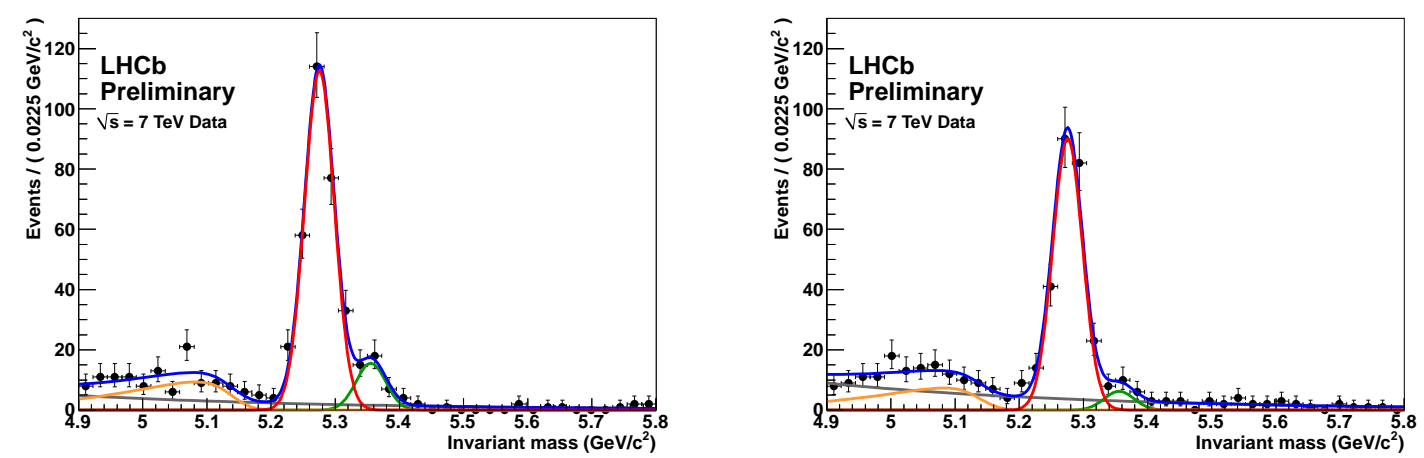

Figure 4: $B^{0}$ and the $B_{s}^{0}$ to $K^{+} \pi^{-}$(left) and $K^{-} \pi^{+}$(right) in $35 \mathrm{pb}^{-1}$ of LHCb data.

$\simeq 0.2$ respectively in $B^{0}$ and $B_{s}^{0}$ decays to $K^{\mp} \pi^{ \pm}$. The corresponding measurement of $C P$ asymmetries would be competitive with CDF results. Again $10^{\circ}-15^{\circ}$ sensitivity to $\gamma$ can be reached with 2011-2012 data.

\section{Conclusion}

First signals from LHCb of hadronic $B$ decays, of interest for the determination of the CKM angle $\gamma$ within tree or loop diagrams, have been shown. This demonstrates the ability of LHCb to study these channels and first results, competitive with current CDF measurements, or first new observations, are expected from more detailed analyses of the 2010 data. The 2011-2012 data taking period, depending on the beam conditions, will potentially allow for $10^{\circ}-15^{\circ}$ sensitivity on $\gamma$ in each class of these decays.

\section{References}

[1] N. Cabibbo, Unitary Symmetry and Leptonic Decays, Phys. Rev. Lett. 10 (1963) 531; M. Kobayashi and T. Maskawa, CP violation in the renormalizable theory of weak interaction, Prog. Theor. Phys. 49 
(1973) 652.

[2] CKMfitter Group (J. Charles and al.), CP Violation and the CKM Matrix: Assessing the Impact of the Asymmetric B Factories, Eur. Phys. J. C41, 1-131 (2005), [hep-ph/0406184], updated results and plots available at: http: / / ckmfitter.in2p3.fr.

[3] M. Ciuchini and al., 2000 CKM-Triangle Analysis A Critical Review with Updated Experimental Inputs and Theoretical Parameters, JHEP 0107 (2001) 013, [hep-ph/ 01230 8], updated results and plots available at : http://www. utfit.org.

[4] J. Zupan, The case for measuring gamma precisely, [hep-ph/1101.0134].

[5] V. Sordini, Measurements of $b \rightarrow u$ amplitude and CKM weak phase $\gamma$ using $B^{0} \rightarrow D^{0} K^{* 0}$ decays reconstructed with the BABAR detector, Ph.D. Thesis, Université de Paris-Sud 11 Orsay and Università di Roma La Sapienza (Jun. 2008).

[6] M. Gronau and D. London, How to determine all the angles of the unitarity triangle from $B_{d}^{0} \rightarrow D K_{\mathrm{S}}^{0}$ and $B_{s}^{0} \rightarrow D \phi$, Phys. Lett. B 253 (1991) 483; M. Gronau and D. Wyler, On determining a weak phase from charged B decay asymmetries, Phys. Lett. B 265 (1991) 172.

[7] D. Atwood, I. Dunietz and A. Soni, Enhanced $C P$ Violation with $B \rightarrow K D^{0}\left(\bar{D}^{0}\right)$ Modes and Extraction of the CKM Angle gamma, Phys. Rev. Lett. 78 (1997) 3257 [hep-ph/9612433]; D. Atwood, I. Dunietz and A. Soni, Improved Methods for Observing CP Violation in $B^{ \pm} \rightarrow K^{ \pm} D^{0}$ and Measuring the CKM Phase gamma, Phys. Rev. D 63 (2001) 036005 [hep-ph/ 0008090 ].

[8] A. Giri, Y. Grossman, A. Soffer and J. Zupan, Determining $\gamma$ using $B^{ \pm} \rightarrow D K^{ \pm}$with multibody $D$ decays, Phys. Rev. D 68 (2003) 054018 [hep-ph/0303187]

[9] R. Aleksan, I. Dunietz and B. Kayser, Determining the CP-violating phase $\gamma$, Z phys C 54 (1992) 653; I. Dunietz and R. Sachs, Asymmetry between inclusive charmed and anticharmed modes in $B^{0}, \bar{B}^{0}$ decay as a measure of CP violation, Phys. Rev. D 37 (1988) 3186 and Erratum: Asymmetry between inclusive charmed and anticharmed modes in $B^{0}, \bar{B}^{0}$ decay as a measure of $C P$ violation, Phys. Rev. $D$ 39 (1989) 3515; I. Dunietz, Clean CKM information from $B_{d} \rightarrow D^{(*) \mp} \pi^{ \pm}$, Phys. Lett. B 427 (1998) 179.

[10] R. Fleischer, New strategies to extract $\beta$ and $\gamma$ from $B^{0} \rightarrow \pi^{+} \pi^{-}$and $B_{s}^{0} \rightarrow K^{+} K^{-}$, Phys. Lett. $B 459$ (1999) 306.

[11] The LHCb Collaboration (B. Adeva and al.), Roadmap for selected key measurements of LHCb , [hep-ex/0912.4179].

[12] The LHCb Collaboration (A. Augusto Alves Jr. and al.), The LHCb detector at the LHC, JINST 3 (2008) S08005, available online at: http://jinst.sissa.it/LHC.

[13] F. Alessio, Operation and Performance of the LHCb Experiment, in these proceedings, PoS (Kruger 2010) 031.

[14] O. Steinkamp, LHCb Status, First Physics and Discovery Potential, in these proceedings, PoS (Kruger 2010) 024.

[15] V. Gligorov, A single track HLT1 trigger, CERN-LHCb-PUB-2011-003.

[16] M. Williams and al., The HLT2 Topological Lines, CERN-LHCb-PUB-2011-002. 